\title{
Cerebrovascular Response to Phenylephrine in Traumatic Brain Injury: A Scoping Systematic Review of the Human and Animal Literature
}

\author{
Logan Froese, ${ }^{1}$ Joshua Dian, ${ }^{2}$ Alwyn Gomez, ${ }^{2,3}$ Bertram Unger, ${ }^{4}$ and Frederick A. Zeiler ${ }^{1-3,5,6, *}$
}

\begin{abstract}
Intravenous phenylephrine (PE) is utilized commonly in critical care for cardiovascular support. Its impact on the cerebrovasculature is unclear and its use may have important implications during states of critical neurological illness. The aim of this study was to perform a scoping review of the literature on the cerebrovascular/cerebral blood flow (CBF) effects of PE in traumatic brain injury (TBI), evaluating both animal models and human studies. We searched MEDLINE, BIOSIS, EMBASE, Global Health, SCOPUS, and the Cochrane Library from inception to January 2020. We identified 12 studies with various animal models and 4 studies in humans with varying TBI pathology. There was a trend toward a consistent increase in mean arterial pressure (MAP) by the injection of PE systemically, and by proxy, an increase of the cerebral perfusion pressure (CPP). There was a consistent constriction of cerebral vessels by PE reported in the small number of studies documenting such a response. However, the heterogeneity of the literature on the $\mathrm{CBF} / \mathrm{cerebral}$ blood volume (CBV) response makes the strength of the conclusions on PE limited. Studies were heterogeneous in design and had significant limitations, with most failing to adjust for confounding factors in cerebrovascular/CBF response. This review highlights the significant knowledge gap on the cerebrovascular/CBF effects of PE administration in $\mathrm{TBI}$, calling for further study on the impact of PE on the cerebrovasculature both in vivo and in experimental settings.
\end{abstract}

Keywords: cerebral blood flow; cerebral blood volume; cerebrovascular reactivity; cerebrovascular response; phenylephrine

\section{Introduction}

1-(3-hydroxyphenyl)- $N$-methylethanolamine or phenylephrine (PE), is an alpha-1 adrenergic drug that is used for systemic blood pressure support in a variety of pathologies. It is one of the most commonly utilized vasopressor agents for cardiovascular support in the management of critically ill patients, through the modulation of adrenergic receptors. ${ }^{1} \mathrm{PE}$ is well known as an effective vasopressor that triggers vascular constriction and increases blood pressure. ${ }^{2}$
Emerging methods of biomedical signal processing for recorded cerebral perfusion pressure (CPP) and intracranial pressure (ICP) monitoring have led to targeted patient therapies, with the goal of improving patient outcomes in traumatic brain injury (TBI) based on continuous measures of cerebrovascular reactivity. ${ }^{3,4}$ Vasopressors, including PE, are used to treat patients with critical neurological illness, such as TBI, with the aim of targeting specific mean arterial pressure (MAP) and CPP goals. ${ }^{1,5}$ However, it remains unclear

\footnotetext{
${ }^{1}$ Biomedical Engineering, ${ }^{2}$ Department of Surgery, ${ }^{3}$ Department of Human Anatomy and Cell Science, ${ }^{4}$ Department of Internal Medicine, ${ }^{5}$ Center on Aging, University of Manitoba, Winnipeg, Manitoba, Canada.

${ }^{6}$ Division of Anesthesia, University of Cambridge, Addenbrooke's Hospital, Cambridge, United Kingdom.
}

*Address correspondence to Frederick A. Zeiler BSc, MD, PhD, CIP, FRCSC, Department of Surgery, Rady Faculty of Health Sciences, University of Manitoba, GF231 Health Sciences Centre, 820 Sherbrook Street, Winnipeg, Manitoba R3A 1R9, Canada, Email: Frederick.Zeiler@umanitoba.ca

(C) Logan Froese et al., 2020; Published by Mary Ann Liebert, Inc. This Open Access article is distributed under the terms of the Creative Commons License (http://creativecommons.org/licenses/by/4.0), which permits unrestricted use, distribution, and reproduction in any medium, provided the original work is properly credited. 
if $\mathrm{PE}$ is detrimental to end-organ cerebral perfusion, or leads to impairment of cerebral autoregulation. Such impacts may have important connotations for outcomes in critically ill patients with TBI, as the effective mediation of cerebral blood flow (CBF) or cerebrovascular reactivity may be vital. ${ }^{6-9}$ As such, understanding the impact of exogenously administered PE on cerebrovascular reactivity and CBF is crucial, as individualized care and personalized targets based on cerebral autoregulation and cerebrovascular response are emerging in management strategies for TBI. ${ }^{8-13}$

The goal of this study was to perform a systematic, scoping review of the available literature on the impact of $\mathrm{PE}$ on cerebrovascular/CBF response in animal models and human subjects with TBI.

\section{Methods}

A systematic review of the literature was conducted using the methodology outlined in the Cochrane Handbook for Systematic Reviewers. ${ }^{14}$ The data were reported in line with the Preferred Reporting Items for Systematic Reviews and Meta-Analyses (PRISMA). ${ }^{15}$ Supplementary Appendix S1 provides the PRISMA checklist.

The review questions and search strategy were decided on by the supervisor (F.A.Z.) and primary author (L.F.).

\section{Search question, population,}

\section{and inclusion/exclusion criteria}

The question posed for systematic review was: What is the effect of exogenous systemically administered $\mathrm{PE}$ on the cerebrovascular response/CBF in TBI? All studies (animal or human), prospective and retrospective, of any size were included.

The primary outcome measure was the impact on objectively measured $\mathrm{CBF}$ or the cerebrovascular responsiveness (i.e., cerebral vasoconstriction or cerebral autoregulation/cerebrovascular reactivity) as documented by: autoradiographic diffusible tracer technique, freely diffusible tracers, thermal diffusion probe, clearance method, laser-Doppler flow probe, flow transducer, flow meter, visual recording software, or any other objective means of CBF determination (including imaging-based techniques). Secondary outcomes included adverse effects of PE administration, including systemic or cerebral end-organ complications.

All studies whether prospective or retrospective, of all sizes, of any age category, and with the use of PE with formal documentation of cerebrovascular response/CBF during administration in the setting of TBI, were eligible for inclusion in this review. Exclusion criteria were: a non-English language study, non-TBI animal model, non-TBI human cohort, or CBF mediation with substance other than PE.

\section{Search strategy}

MEDLINE, BIOSIS, EMBASE, Global Health, SCOPUS, and the Cochrane Library from inception to January 2020 were searched using individualized search strategies for each database. The search strategy for MEDLINE can be reviewed in Supplementary Appendix S2; a similar search strategy was used for the other databases. In addition, the reference lists of reviewed articles on the cerebral blood vessels/CBF response to $\mathrm{PE}$ were examined to ensure no references were left out.

\section{Study selection}

Using two reviewers (L.F. and J.D.), a two-step review of all articles returned by our search strategy was performed. First, the reviewers independently screened all titles and abstracts of the returned articles to decide whether they met the inclusion criteria. Second, full text of each returned article was assessed to confirm whether it met the inclusion criteria and that the primary outcome of cerebrovascular/CBF response to PE was documented. Third, the attained literature was separated into TBI versus non-TBI models. Any discrepancies between the two reviewers were resolved by a third party (F.A.Z.).

\section{Data collection}

Data were extracted from the selected articles and stored in multiple electronic databases to ensure data integrity.

\section{Animal studies}

Data abstraction fields included the following: number of animals, type of study, animal model characteristics, the goal of the study, type of vasopressors administered, dose of vasopressors administered, technique of $\mathrm{CBF} /$ vasculature assessment, cerebrovascular/CBF response to PE, other outcomes, and general conclusions.

\section{Human studies}

Data fields included the following: number of patients, study type, article location, mean age, patient characteristics, goal of the study, dose, dose duration, technique to measure cerebrovascular/CBF response, the documented cerebrovascular/CBF response, other outcomes, and conclusion.

\section{Statistical analysis}

A meta-analysis was not performed in this study because of the heterogeneity of model types, study designs, and data. 


\section{Results}

Search results and study characteristics

The results of the search strategy across all databases and other sources are summarized in Figure 1. Overall, 2315 articles were identified from the databases searched. Removed were 1246 articles because of duplicate references, leaving 1069 to review. By applying the inclusion/exclusion criteria to the title and abstract of these articles, we identified 261 articles that fit these criteria. No articles were added from reference sections of pertinent review articles, leaving a total of 261 articles to review. On applying the inclusion/exclusion criteria to the full-text documents, only 16 articles were found eligible for inclusion in the systematic review, all from the database search. Articles were excluded because they either did not report details around objectively measured cerebrovascular/CBF response to $\mathrm{PE}$ administration, were review articles, were non-relevant, or contained non-TBI cohorts.

\section{Part 1: TBI animal models}

Tables 1 and 2 outline the animal TBI study characteristics and cerebrovascular/CBF responses, respectively. Of the 12 articles that had animal models with TBI, the following models were described: induced fluid percussion injury, ${ }^{16-22}$ impact-acceleration injury, ${ }^{23}$ rapid

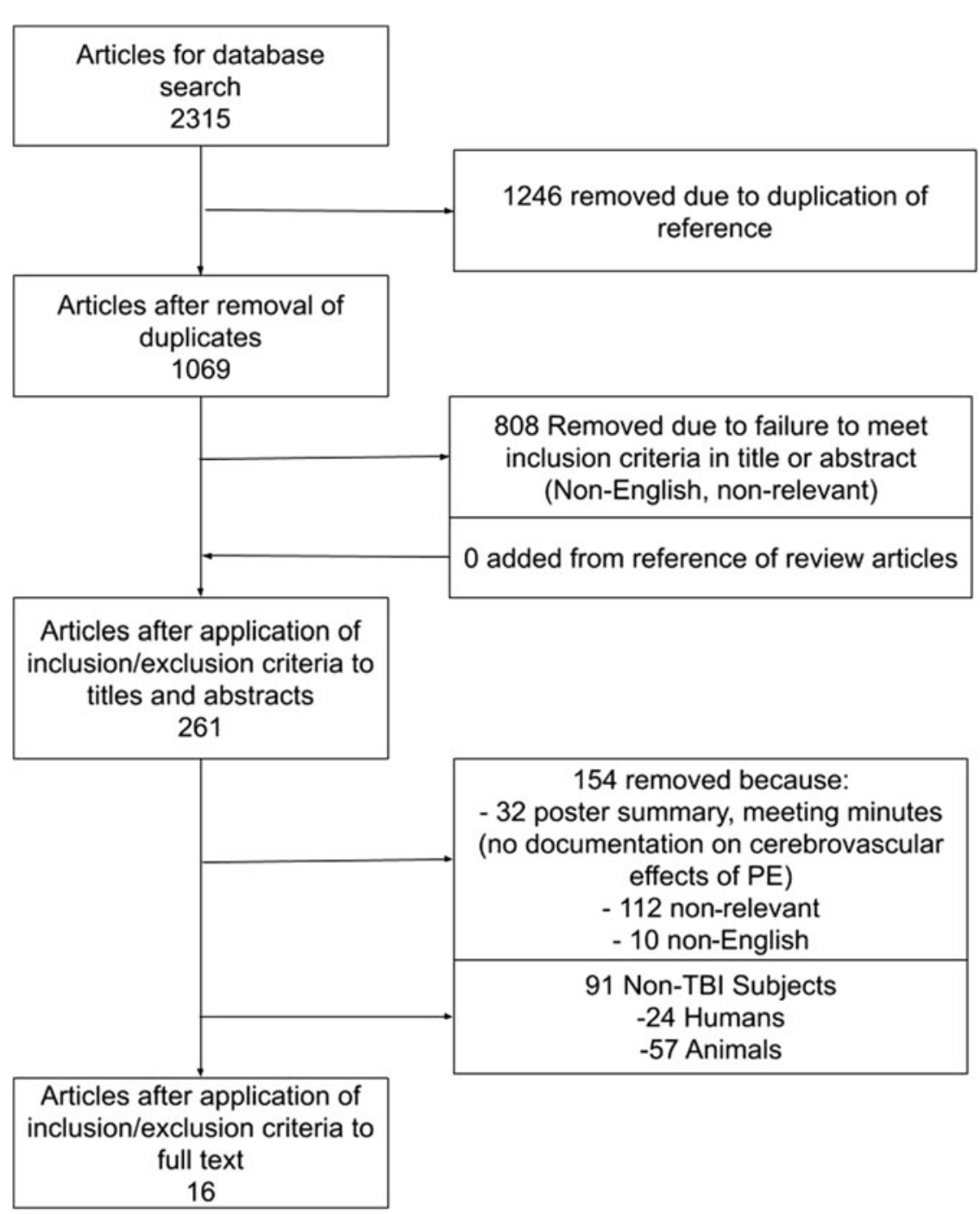

FIG. 1. PRISMA (Preferred Reporting in Systematic Reviews and Meta-Analysis) flow diagram of search results and filtering. 


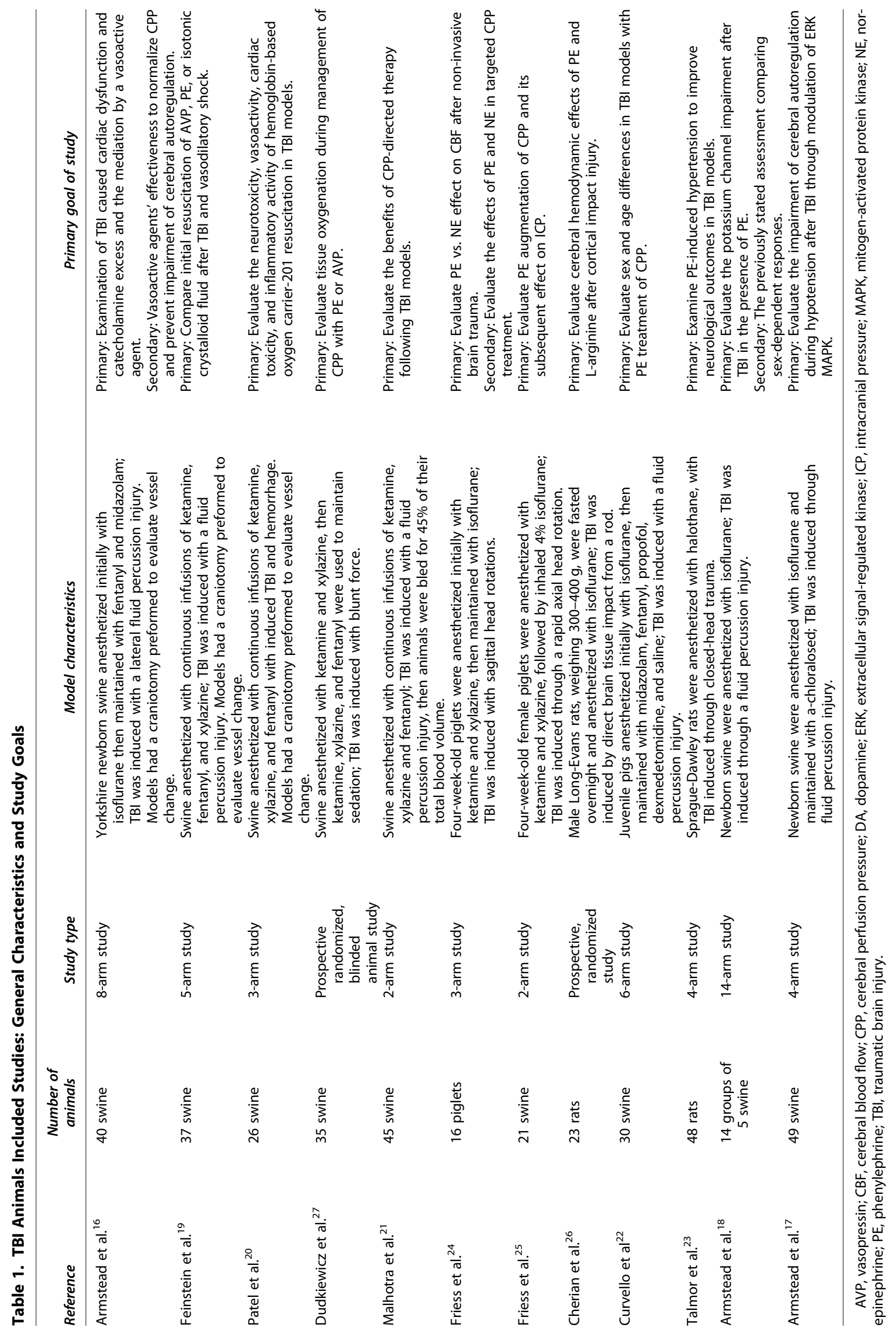




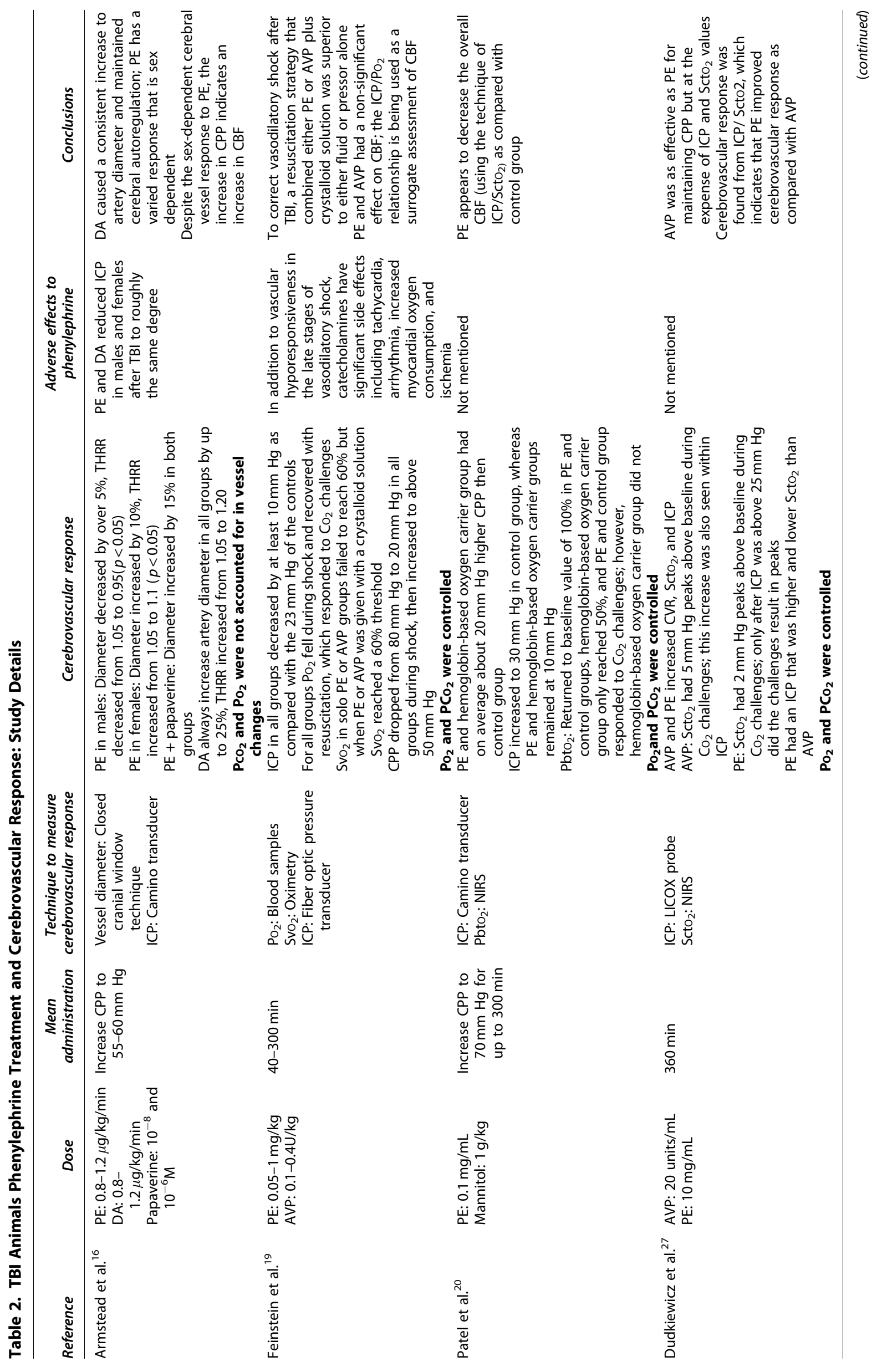




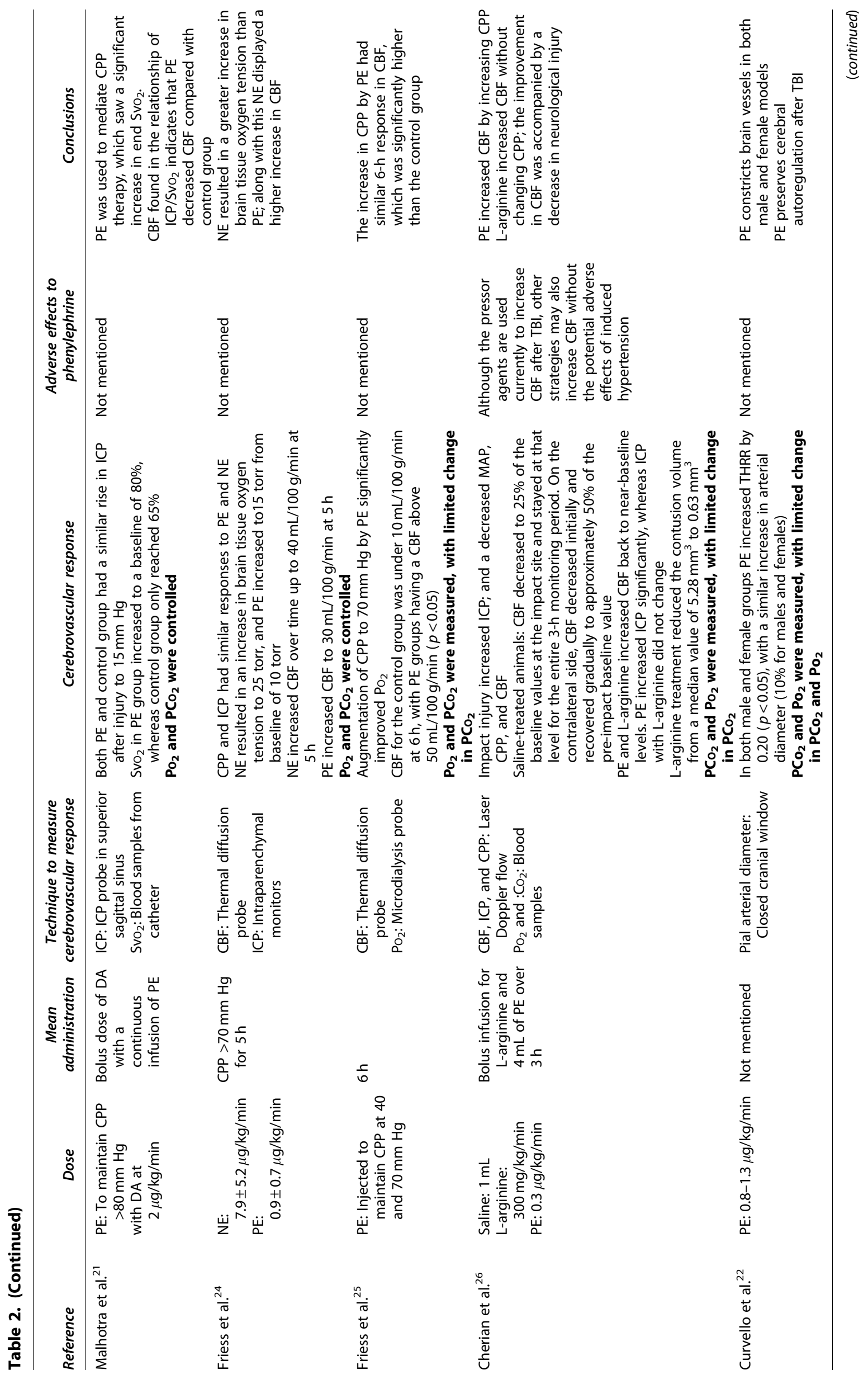




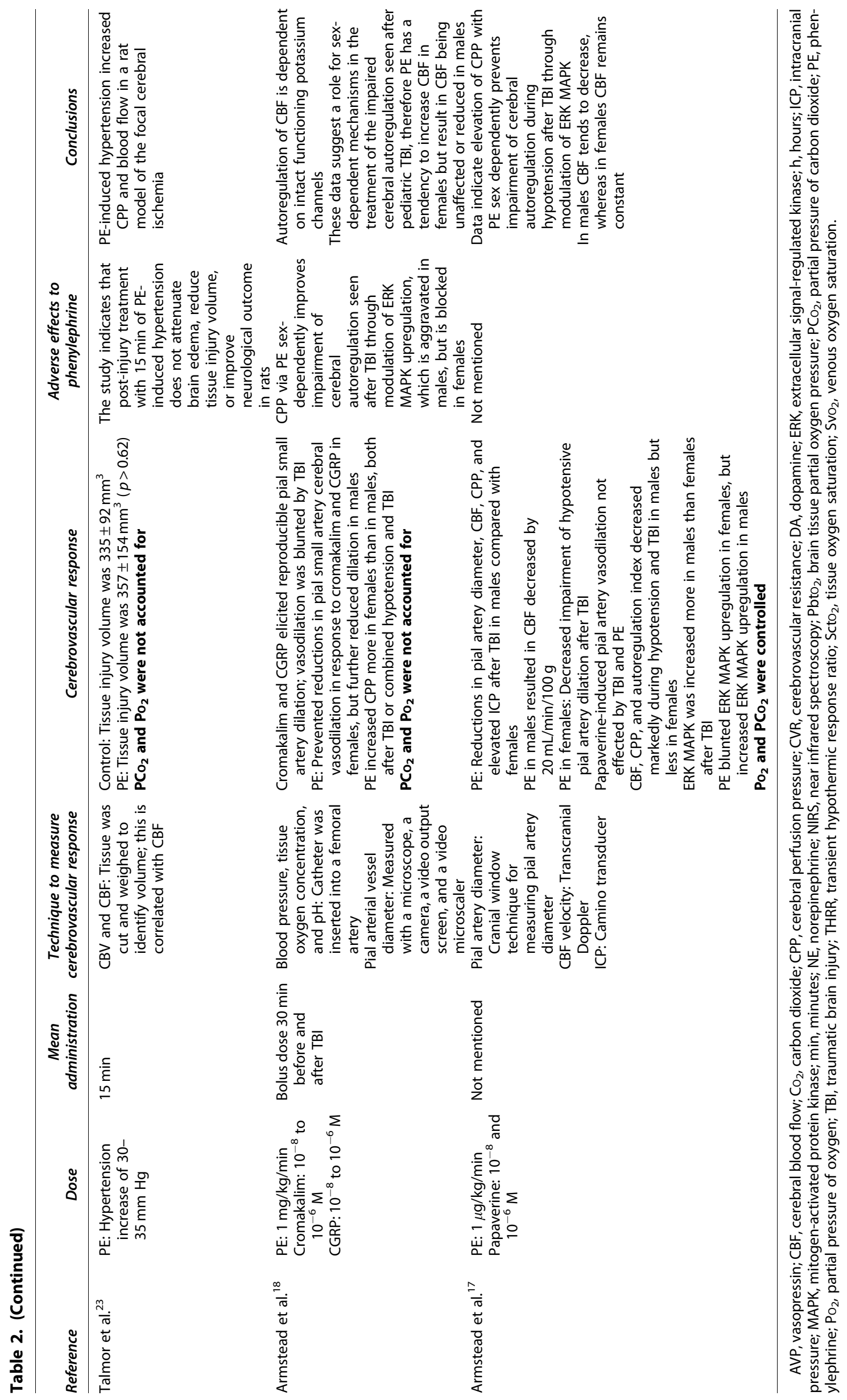


axial head rotation, ${ }^{24,25}$ and rod directed into the parietal cortex. ${ }^{26,27}$ To measure cerebrovascular/CBF response, a variety of techniques were used including: comparing ICP (found with strain gauge or catheter transducer-a surrogate metric for pulsatile cerebral blood volume [CBV]) and partial or region oxygenation (found with near infrared spectroscopy or blood samples), thermal diffusion probes, cortical laser Doppler flowmetry and direct measurement of vasculature change, or change in cerebral tissue weight (i.e., as a metric of $\mathrm{CBV}$ ). There were 10 studies that used swine models and 2 that used rats. ${ }^{23,26}$ Of the swine model studies, 5 were conducted on newborn pigs, ${ }^{16-18,24,25}$ one had juvenile models, ${ }^{22}$ and the remaining 4 did not specify age (but were assumed to be pre-adult based on weight). ${ }^{19-21,27}$ The majority of studies controlled for both $\mathrm{PO}_{2}$ and $\mathrm{PCO}_{2}$, while administering heavy sedation.

$\mathrm{CBF} / \mathrm{CBV}$ response. A clear and consistent increase in CPP was noted in all studies in which subjects had PE injected systemically. ${ }^{16,19-22,24-27}$ However this change did not always translate to an increase in CBF or ICP (a surrogate measure of CBV), shown by the lack of impact on ICP and CBF in two studies. ${ }^{21,25}$ In the studies in which cerebral autoregulation was impaired, there was a significant increase in ICP and CBF caused by $\mathrm{PE}$ injection (cerebral autoregulation was impaired with a fluid percussion injury). ${ }^{16-18,22}$ These studies evaluated the CBF before and after injury, as well as before and after PE injection, with $\mathrm{CBF}$ increasing toward $^{16}$ or exceeding baseline values post-injection. ${ }^{17,18,22}$ One study evaluated cerebral autoregulation (direct visual change in pial blood vessels) during PE injections, with cerebral autoregulation becoming impaired in male piglets only. ${ }^{16}$ Further, in the setting of a traumatic impact (rod injected to a deformation of $3 \mathrm{~mm}$ in parietal cortex) with sodium nitroprusside infusion, a decrease in CBF and ICP occurred, which was then reversed with $\mathrm{PE}$ injection back to near baseline values. $^{26}$ In a study utilizing systemic injection of PE, and that measured brain tissue mass change, there was a direct increase in tissue volume mass (correlated with $\mathrm{CBV}){ }^{23}$

Direct effect on cerebral vessels. The studies that measured the direct effect of PE on cerebral vessels were all neonatal models. Similar effects of PE in these studies were shown within cerebral vessels, with all studies that measured cerebrovascular diameter or tension demonstrating cerebral vasoconstriction. In two studies, PE was injected directly through a cranial window on the vessels, demonstrating a reduction in pial artery diameters. ${ }^{17,22}$ One study that used the cranial window technique, but utilized systemic injection of PE, found mixed results to vessel change. ${ }^{16}$ Another study found that pial arterial diameter decreases in male piglets during systemic $\mathrm{PE}$ injection, but increases in female piglets. ${ }^{18}$

\section{Part 2: TBI human models}

Tables 3 and 4 outline the human TBI study characteristics and cerebrovascular/CBF responses, respectively. There are four studies with human patients with TBI who were injected with $\mathrm{PE}$, and had CBF or cerebrovascular responses measured. ${ }^{28-31}$ One study did not specify the ages of the patients, who had a Glasgow Coma Scale (GCS) score $\leq 8 .^{28}$ The other three studies all had patients with mean ages of 30-35 years. Of these three studies, one had patients with a GCS score between 3 and $14,{ }^{29}$ one had patients with diffuse brain injury and a GCS score $\leq 12,{ }^{30}$ and one had patients with a mean GCS score of 5.5 and an injury severity score of $37 . .^{31}$ In these studies, CBF was measured with the ${ }^{133}$ Xenon inhalation method, ${ }^{28,29}$ the arterial jugular difference in oxygen, ${ }^{30}$ or computed tomography (CT) perfusion. ${ }^{31}$ Most studies adjusted for $\mathrm{PCO}_{2}$ but failed to mention whether $\mathrm{PO}_{2}$ was controlled. Sedative regiments for these studies were not clearly indicated.

CBF/CBV response. All studies objectively documented the CBF response to PE injection in human patients with TBI. One study demonstrated an increase in $\mathrm{CPP}$, with concordant MAP increase. ${ }^{30}$ The CBF response in this study varied and was inconsistent across the population, demonstrating both increases and decreases. A second study failed to demonstrate a significant change in CBF or ICP (i.e., surrogate of pulsatile $\mathrm{CBV}) .{ }^{31} \mathrm{~A}$ third study compared patients with intact versus impaired cerebrovascular reactivity (measured by the percentage change in $\mathrm{CPP} / \mathrm{CBF}$ with the clearance method) and found that PE substantially increased CBF, by up to 53\%, when regulation was impaired versus not impaired. ${ }^{28}$ Regarding the CBV response to PE administration, an effect on ICP was also shown, with those displaying impaired cerebrovascular reactivity having a higher mean change in ICP of $3.8 \mathrm{~mm} \mathrm{Hg}$ versus $1.5 \mathrm{~mm} \mathrm{Hg}$ to PE injection, for the impaired versus intact groups, respectively 


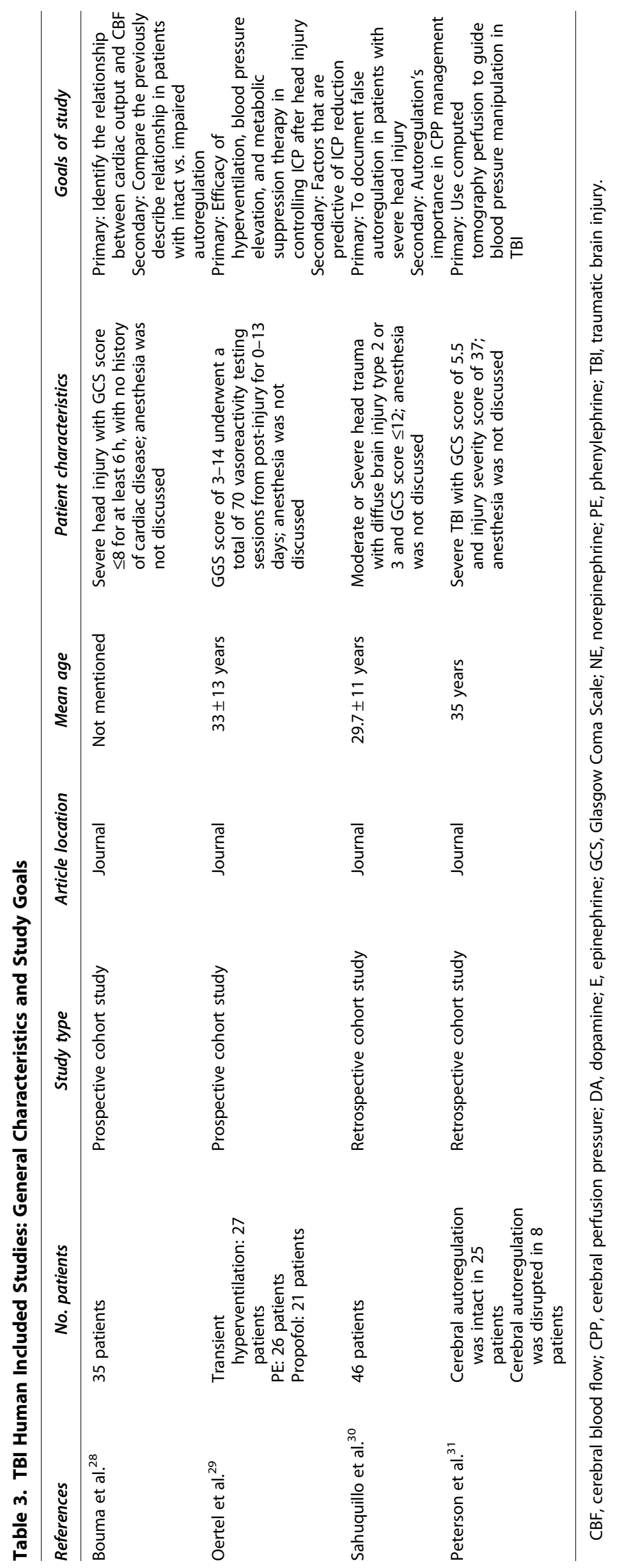




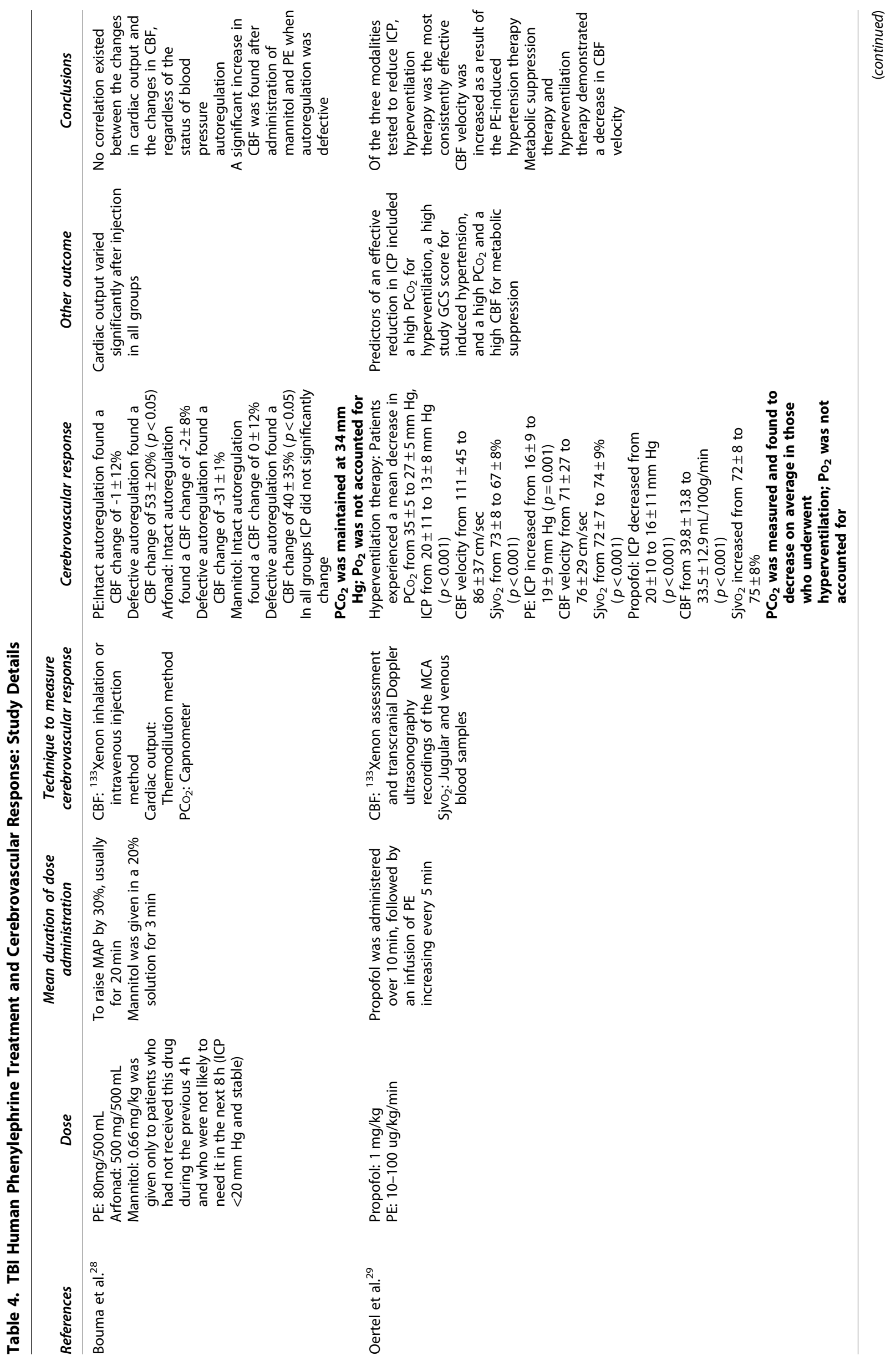




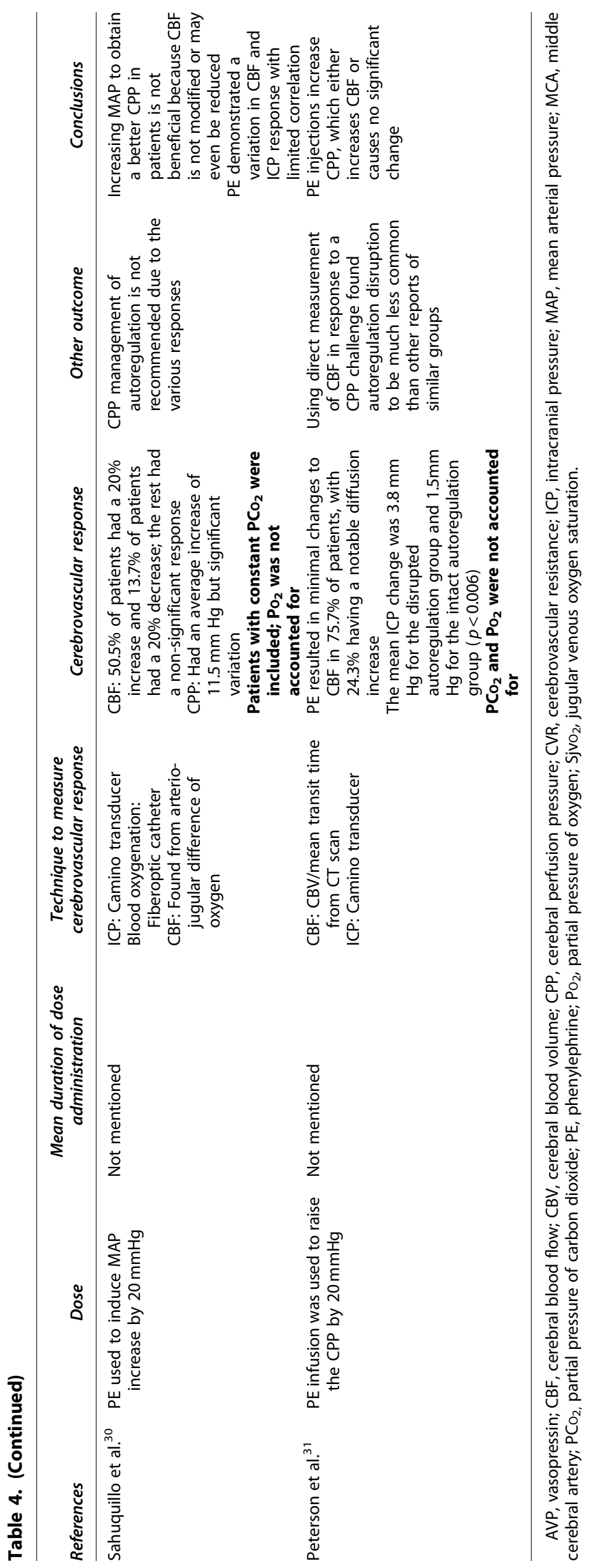


(cerebrovascular reactivity was consider intact if an increase in CPP did not change CBF as measured by CT perfusion). ${ }^{31}$

Cerebrovascular response. No human study measured the direct cerebrovascular response to PE in human subjects. Despite this, there were some studies that attempted to quantify cerebrovascular resistance (CVR) from the $\mathrm{CPP} / \mathrm{CBF}$ relationship. ${ }^{28,30}$ However, there was no consensus on the CVR response to PE administration, with CVR demonstrating a variable response.

Adverse events to phenylephrine in all models

The adverse effects of PE in animal models included: bradycardia, vascular hyperresponsiveness in the late stages of vasodilatory shock, tachycardia, arrhythmia, increased myocardial oxygen consumption, and ischemia. ${ }^{19,23}$ One study showed an increased expression of cerebral V1 receptor subtype after TBI, and the subsequent formation of cerebral edema. ${ }^{19}$ In two studies, one animal and one human, cerebral autoregulation was impaired with PE administration (i.e., there was an in-phase direct increase in $\mathrm{CBF}$ with increased MAP) ${ }^{17,28}$ Impaired autoregulation appeared to demonstrate a sex predominance in another study, which found PE induced impaired autoregulation preferentially in male neonatal pigs, as compared with females. ${ }^{17}$

\section{Discussion}

PE is a commonly used vasopressor agent to increase MAP in a variety of treatment practices. In critical care patients, and specifically those with TBI, PE may be utilized in targeting of ICP, CPP (and hopefully $\mathrm{CBF}$ ) to improve outcomes. Knowledge of the cerebrovascular response to various commonly utilized vasopressor agents, such as $\mathrm{PE}$, is crucial for the provision of effective treatments in TBI. ${ }^{4,6-11,13,32,33}$ The current literature on the cerebrovascular effects of PE leaves uncertainty in predicting the cerebrovascular/CBF response to its administration. Through our scoping review we attempted to gather all studies that documented the administration of $\mathrm{PE}$ and measured its effects on the cerebrovascular response/CBF, in both animal models and human subjects afflicted with TBI. Some important points and preliminary trends can be gleaned from this review, although it must be acknowledged that, overall, the literature in this area is limited, highlighting a significant knowledge gap.
First, PE injection leads to a direct increase in MAP, and subsequently CPP. MAP increase is reported in all studies regardless of design or method, and subsequently led to a CPP increase in all studies that concurrently evaluated ICP. This general finding is not surprising, as the main role for PE in critical neurological illness is the augmentation of MAP and CPP. Particularly, in the treatment of moderate/severe TBI, this aspect of $\mathrm{PE}$ is attractive and is employed to maintain ICP/CPP targets, as suggested by current guidelinebased therapies. ${ }^{4,34}$ However, the exact dose-response relationship between MAP and CPP was not clearly delineated in many studies, particularly in the human studies. As we transition to personalized physiological targets in TBI care, such as optimal CPP, ${ }^{10,11,13}$ we may find ourselves targeting CPP values well above the current Brain Trauma Foundation defined target range of $60-70 \mathrm{~mm} \mathrm{Hg}{ }^{4}$ As such, with increasing $\mathrm{PE}$ dosing, the relationship between PE dose, MAP, and subsequently CPP may prove to be non-linear. In fact, with escalating doses of $\mathrm{PE}$ and the subsequent increase in MAP, there is the potential to overwhelm cerebrovascular reactivity and cause degeneration of the classic Lassen autoregulatory curve. ${ }^{35}$ This would expose patients to significant secondary injury, and poor long-term outcomes, as reported in the human TBI literature. ${ }^{7,33,36-38}$ The current literature in both animal and human TBI fails to inform whether such an upper limit of PE dosing exists. It is likely the specific dose-response is dependent on a variety of individual factors, including genotype. ${ }^{39}$ Thus, despite the similar results seen with $\mathrm{PE}$ administration in both animal and human TBI studies, a true understanding of its impact on the cerebral vasculature remains limited, necessitating future investigation.

Second, there were a limited number of TBI animal model studies that measured directed cerebral vessel response, with only four studies in neonate piglet models. ${ }^{16-18,22}$ The literature lacks homogeneity in model type and study design. However, PE administration demonstrated consistent constriction of the cerebral vessels. It must be acknowledged though that the ability to translate cerebrovascular responses to PE in immature neonatal vessels to mature adult cerebral vessels is limited. Despite the clear limitations of these data, there is sufficient homogeneity in the outcome to suggest that PE has a direct vasoconstrictive effect on the cerebral pre-capillary arterioles. This suggested effect is important; given the strong independent association between impaired cerebrovascular reactivity and poor 
patient outcome in TBI, ${ }^{37,38}$ it is clear that cerebrovascular reactivity is an important aspect of cerebral physiology that impacts secondary injury exposure and outcome. Administration of vasoactive compounds such as PE may directly impact cerebrovascular reactivity, given cerebrovascular reactivity is believed to occur at the pre-capillary arteriole level..$^{35,40-42}$ As highlighted in this review, literature on an exact dose-response at the pre-capillary arteriole and its impact on continuously measured cerebrovascular reactivity is absent. Therefore the impact of continuous PE dosing on cerebrovascular reactivity in TBI remains unclear, supporting the need for future research.

Third, despite the general increase in CPP, the quantitative CBF response in human models was underinvestigated. Of the two studies that showed a $\mathrm{CBF}$ increase with $\mathrm{PE}$ injection, either autoregulation was impaired, ${ }^{28}$ or there was a conflicting increase and subsequent decrease in $\mathrm{CBF}^{30}$ The animal literature also demonstrated an increase in CBF. However, in the study design PE was never the sole focus of the study, with other medications co-administered, leading to significant confounding. Without a true understanding of the impact of $\mathrm{PE}$ on the pre-capillary vascular response, and subsequently $\mathrm{CBF}$, we remain in the dark regarding safe dosing ranges in TBI.

Finally, within the literature it is unclear what the impact of systemic PE injection is on cerebrovascular reactivity/cerebral autoregulation in animal/human TBI, as there were no studies identified that evaluated the cerebral microvascular change within human models. Thus, it remains unknown if there exists a dose-response effect on cerebral autoregulation, where specific $\mathrm{PE}$ dose ranges may lead to impaired autoregulation. The animal literature adds insight to the effects of $\mathrm{PE}$ on cerebral vessels as reported in four studies in which PE injected systemically or locally found vessels to constrict. ${ }^{16-18,22}$ Further, one animal study suggested a potential disparity in autoregulatory response to $\mathrm{PE}$ based on sex, with males displaying deterioration in cerebral autoregulation caused by PE injection. ${ }^{17}$ However, the lack of direct translation of these data to human models and patient care leaves limitations to be addressed in future studies. Also, there was a lack of consistency in evaluating cerebral autoregulation. Within the human studies alone, there were three different methods used to evaluate if cerebral autoregulation was intact. A refined and accurate way to quantify cerebral autoregulation in vivo is needed. Such autoregulatory monitoring should take the form of commonly adopted continuous measures of cerebrovascular reactivity, such as pressure reactivity index (PRx).

Thus, overall, given the outlined trends and limitations of the identified literature in this area, we are left with an inconclusive view of the impact of PE on $\mathrm{CBF} /$ cerebrovasculature in TBI. But a "negative" or "inconclusive" systematic review does provide important information regarding the current gap in knowledge. Despite PE being a commonly administered vasoactive compound in neurocritical care, we have a limited idea of its impact on the end organ of our primary interest, the brain. Aside from the general increase in MAP and trend toward an increase in CVR, when measured, the impact on cerebral autoregulation/cerebrovascular reactivity and $\mathrm{CBF}$ is unclear. Such a glaring hole in the available TBI literature is both important to identify/quantify-which we believe this review has done-and represents an area where future research is of utmost importance (see "Future directions" section for more details). As such, despite the limited conclusions drawn here, we believe highlighting such limitations/deficiencies will lead to future directed study of the cerebral impacts of PE.

\section{Limitations}

Although this review is comprehensive, there exist significant limitations. First, the overall literature was very heterogeneous in design. This limits how definitive we can be in our statements regarding the impact of PE on $\mathrm{CBF}$, and cerebrovascular response in TBI. Similarly, there was heterogeneity in the co-administration of vasoactive compounds, sedative regimens, and documentation of $\mathrm{PCO}_{2}$ and $\mathrm{PO}_{2}$ levels during measurement of $\mathrm{CBF} /$ cerebrovascular response. Also, the heterogeneity in $\mathrm{CBF} /$ cerebrovascular response measurement techniques between studies further limits our ability to consolidate findings between studies. Thus, the conclusions regarding the impact of $\mathrm{PE}$ on $\mathrm{CBF} /$ cerebrovascular response lack strength. Second, animal models do not necessarily translate directly to human response to PE. Therefore, despite a larger volume of literature on animal TBI models, it remains unknown if any of the data can directly translate to human patients. Similarly, some models focused on young or neonatal animals, in which it is known that the cerebrovasculature is in an immature state. The response of such vessels to PE administration may not reflect the true response in mature animals, or adult humans. As such, we are again limited in our ability to extrapolate results. Third, the disparity in response shown in $\mathrm{CBF}$ to $\mathrm{PE}$ 
administration limits our ability to confidently state the impact of PE on CBF, both in animals and humans afflicted with TBI. This is further clouded by the different populations and models studied. Finally, almost all studies identified, both animal and human, failed to focus on the cerebrovascular response of the precapillary arterioles to PE administration. The animal studies that did utilized neonatal models. These precapillary arterioles, which are believed to be the major players in cerebral autoregulatory response, may behave differently in neonates as compared with adults. Knowledge of the impact of PE on such vessels is crucial, as impaired cerebrovascular function is a known associate of poor outcome in the setting of TBI.

\section{Future directions}

Through this review of the literature on the effect of $\mathrm{PE}$ on $\mathrm{CBF} /$ cerebral vessel response, we found a trend toward an increase in MAP, CPP, and CVR across all animal models and human subjects with TBI. However, the $\mathrm{CBF} /$ cerebrovascular response remains unclear, given the disparity in responses shown. Despite the limited concrete conclusions on the impact of PE on TBI derived, this review has highlighted an important knowledge gap related to a readily administered vasoactive compound in neurocritical care, and we have outlined future avenues that require investigation.

Future work on PE and the cerebrovascular response should focus on the dose response in both animals and humans, evaluating regional disparities in vascular response across the age spectrum and between sex groups, and on the pre-capillary arterioles responsible for cerebral autoregulation. Human TBI research would benefit from multi-center and comprehensive high-frequency physiological data sets. Such data would include accurate and complete treatment markups linked to high-frequency physiology, allowing assessment of the impact of PE administration/dose changes on various aspects of cerebral physiology. The high-frequency physiology data sets would need to comprise full waveform data, allowing for advanced bio-signal analytics, and derivation of continuous measures of cerebrovascular reactivity and compensatory reserve. Similarly, the monitoring modalities employed in this human TBI population would ideally include not only ICP/ICP-derived metrics, but also measures of end-organ $\mathrm{CBF}$ (such as regional thermal diffusion probes or multi-channel near infrared diffuse correlation/time-resolved spectroscopy), end-organ oxygen delivery (through brain tissue oxygen probes or near infrared spectroscopy), and cerebral metabolism (through microdialysis). Further, with all monitoring in place, manipulations in PE dosing would need to occur, to ensure a comprehensive understanding of the impact of changes in $\mathrm{PE}$ on cerebrovascular physiology in vivo.

We envision the experimental animal model work employing both healthy controls and TBI models. An important aspect of such future investigations would be controlling/adjusting for potential confounders. This would require uniform sedation regimens and accurate control of $\mathrm{PCO}_{2} / \mathrm{PO}_{2}$. Evaluation of $\mathrm{CBF} /$ cerebrovascular response in such animal models would benefit from continuous high-temporal frequency methods, using clinically applied multi-modal monitoring/signal processing techniques. Such monitoring could, in theory, deploy multiple monitors simultaneously, assessing regional $\mathrm{CBF}$, cerebrovascular reactivity, oxygen delivery (using brain tissue oxygen probes or advance near infrared spectroscopy monitoring), and metabolics (using microdialysis). Within the TBI models, given regional disruption in blood-brain barrier integrity, such high-temporal and spatial continuous monitoring is crucial, as regional disparities in $\mathrm{CBF} /$ cerebrovascular response post-TBI may be driven by regional differences in blood-brain barrier functionality. This research should involve the application of multi-modal cerebral physiological monitoring, and the derivation of continuous metrics of cerebrovascular reactivity.

Finally, both human and animal research would benefit from concordant genotyping and assessment of vascular protein/vasoactive small-molecule biomarkers associated with cerebrovascular control. ${ }^{43}$ The outlined multi-faceted, comprehensive approach to monitoring is the only way to improve understanding of the impact of $\mathrm{PE}$ administration on $\mathrm{CBF} /$ cerebrovascular response in TBI. Such investigations are the ongoing aspirations/work of various European, ${ }^{44}$ North American, ${ }^{45-50}$ and Canadian ${ }^{51}$ collaborative efforts.

\section{Conclusion}

In TBI, the human and animal literature on the cerebrovascular/CBF effects of $\mathrm{PE}$ is scarce and significantly limited. Limitations include study and model design heterogeneity and failing to adjust for potential confounders. The identified literature demonstrated a trend toward increased MAP, CPP, and CVR with PE administration. The direct cerebrovascular response 
to $\mathrm{PE}$ administration demonstrated cerebral vasoconstriction in TBI animal models. However, the CBF response to $\mathrm{PE}$ administration was heterogeneous, in both animal and human studies identified, with no definitive conclusions. This review highlights a major knowledge gap regarding the impact of PE in TBI. Further research into the $\mathrm{CBF}$ and pre-capillary arteriole response to $\mathrm{PE}$ administration in TBI is required, as it carries important implications for treatment in moderate/severe TBI.

\section{Acknowledgments}

F.A.Z. receives research support from the Manitoba Public Insurance (MPR) Neuroscience/TBI Research Endowment, the Health Sciences Center Foundation Winnipeg, the United States National Institutes of Health (NIH) through the National Institute of Neurological Disorders and Stroke (NINDS), the Canadian Institutes of Health Research (CIHR), the Canada Foundation for Innovation (CFI), Research Manitoba, the University of Manitoba VPRI Research Investment Fund (RIF), the University of Manitoba Center on Aging, and the University of Manitoba Rudy Falk Clinician-Scientist Professorship. A.G. is supported through the University of Manitoba Clinician Investigator Program.

\section{Funding Information}

This work was supported directly through the University of Manitoba, Department of Surgery GFT Research Grant (L.F.), and the University of Manitoba Office of Research Services (ORS), University Research Grant Program (URGP; L.F.).

\section{Author Disclosure Information}

No competing financial interests exist.

\section{Supplementary Material}

Supplementary Appendix S1

Supplementary Appendix S2

\section{References}

1. Kellum, J.A., and Pinsky, M.R. (2002). Use of vasopressor agents in critically ill patients. Curr. Opin. Crit. Care 8, 236.

2. Hollenberg, S.M. (2011). Vasoactive drugs in circulatory shock. Am. J. Respir. Crit. Care Med. 183, 847-855.

3. Kochanek, P.M., Tasker, R.C., Bell, M.J., Adelson, P.D., Carney, N., VaviIala, M.S., Selden, N.R., Bratton, S.L., Grant, G.A., Kissoon, N., Reuter-Rice, K.E., and Wainwright, M.S. (2019). Management of Pediatric Severe Traumatic Brain Injury: 2019 Consensus and Guidelines-Based Algorithm for First and Second Tier Therapies. Pediatr. Crit. Care Med. 20, 269-279.
4. Carney, N., Totten, A.M., O'Reilly, C., Ullman, J.S., Hawryluk, G.W.J., Bell, M.J., Bratton, S.L., Chesnut, R., Harris, O.A., Kissoon, N., Rubiano, A.M., Shutter, L., Tasker, R.C., Vavilala, M.S., Wilberger, J., Wright, D.W., and Ghajar, J. (2017). Guidelines for the Management of Severe Traumatic Brain Injury, Fourth Edition. Neurosurgery 80, 6-15.

5. Auchet, T., Regnier, M.-A., Girerd, N., and Levy, B. (2017). Outcome of patients with septic shock and high-dose vasopressor therapy. Ann. Intensive Care 7, doi: 10.1186/s13613-017-0261-x.

6. Budohoski, K.P., Czosnyka, M., Kirkpatrick, P.J., Smielewski, P., Steiner, L.A., and Pickard, J.D. (2013). Clinical relevance of cerebral autoregulation following subarachnoid haemorrhage. Nat. Rev. Neurol. 9, 152-163.

7. Czosnyka, M., Smielewski, P., Kirkpatrick, P., Laing, R.J., Menon, D., and Pickard, J.D. (1997). Continuous assessment of the cerebral vasomotor reactivity in head injury. Neurosurgery 41, 11-19.

8. Zeiler, F.A., Ercole, A., Cabeleira, M., Beqiri, E., Zoerle, T., Carbonara, M., Stocchetti, N., Menon, D.K., Lazaridis, C., Smielewski, P., Czosnyka, M., and CENTER-TBI High Resolution ICU Sub-Study Participants and Investigators. (2020). Patient-specific ICP Epidemiologic Thresholds in Adult Traumatic Brain Injury: A CENTER-TBI validation study. J. Neurosurg. Anesthesiol. doi: 10.1097/ANA.0000000000000616 [Epub ahead of print].

9. Lazaridis, C., DeSantis, S.M., Smielewski, P., Menon, D.K., Hutchinson, P., Pickard, J.D., and Czosnyka, M. (2014). Patient-specific thresholds of intracranial pressure in severe traumatic brain injury. J. Neurosurg. 120, 893-900.

10. Steiner, L., Czosnyka, M., Piechnik, S., Smielewski, P., Chatfield, D., Menon, D., and Pickard, J. (2002). Continuous monitoring of cerebrovascular pressure reactivity allows determination of optimal cerebral perfusion pressure in patients with traumatic brain injury. Crit. Care Med. 30, 733-738.

11. Aries, M.J., Czosnyka, M., Budohoski, K., Steiner, L., Lavinio, A., Kolias, A., Hutchinson, P., Brady, K., Menon, D., Pickard, J., and Smielewski, P. (2012). Continuous determination of optimal cerebral perfusion pressure in traumatic brain injury. Crit. Care Med. 40, 2456-2463.

12. Zeiler, F.A., Ercole, A., Cabeleira, M., Carbonara, M., Stocchetti, N., Menon, D.K., Smielewski, P., Czosnyka, M., Anke, A., Beer, R., Bellander, B.-M., Buki, A., Chevallard, G., Chieregato, A., Citerio, G., Czeiter, E., Depreitere, B., Eapen, G., Frisvold, S., Helbok, R., Jankowski, S., Kondziella, D., Koskinen, L.-O., Meyfroidt, G., Moeller, K., Nelson, D., Piippo-Karjalainen, A., Radoi, A., Ragauskas, A., Raj, R., Rhodes, J., Rocka, S., Rossaint, R., Sahuquillo, J., Sakowitz, O., Stevanovic, A., Sundström, N., Takala, R., Tamosuitis, T., Tenovuo, O., Vajkoczy, P., Vargiolu, A., Vilcinis, R., Wolf, S., and Younsi, A. (2018). Comparison of Performance of Different Optimal Cerebral Perfusion Pressure Parameters for Outcome Prediction in Adult Traumatic Brain Injury: a Collaborative European NeuroTrauma Effectiveness Research in Traumatic Brain Injury (CENTER-TBI) study. J. Neurotrauma 36, 1505-1517.

13. Beqiri, E., Smielewski, P., Robba, C., Czosnyka, M., Cabeleira, M.T., Tas, J. Donnelly, J., Outtrim, J.G., Hutchinson, P., Menon, D., Meyfroidt, G., Depreitere, B., Aries, M.J., and Ercole, A. (2019). Feasibility of individualised severe traumatic brain injury management using an automated assessment of optimal cerebral perfusion pressure: the COGiTATE phase II study protocol. BMJ Open 9, e030727.

14. Higgins, J., and Thomas, J. (2019). Cochrane Handbook for Systematic Reviews of Interventions. www.training.cochrane.org/handbook/current (Last accessed January 5, 2020).

15. Moher, D., Liberati, A., and Tetzlaff, J. (2009). Preferred reporting items for systematic reviews and meta-analysis: the PRISMA statement. Ann. Intern. Med. 151, 264-269.

16. Armstead, W.M., and Vavilala, M.S. (2019). Cerebral perfusion pressure directed-therapy modulates cardiac dysfunction after traumatic brain injury to influence cerebral autoregulation in pigs. Neurocrit. Care 31, 476-485.

17. Armstead, W.M., Kiessling, J.W., Kofke, W.A., and Vavilala, M.S. (2010). Impaired cerebral blood flow autoregulation during posttraumatic arterial hypotension after fluid percussion brain injury is prevented by phenylephrine in female but exacerbated in male piglets by extracellular signal-related kinase mitogen-activated protein kinase upregulation. Crit. Care Med. 38, 1868-1874.

18. Armstead, W.M., Kiessling, J.W., Riley, J., Kofke, W.A., and Vavilala, M.S. (2011). Phenylephrine infusion prevents impairment of ATP- and calcium- 
sensitive potassium channel-mediated cerebrovasodilation after brain injury in female, but aggravates impairment in male, piglets through modulation of ERK MAPK upregulation. J. Neurotrauma 28, 105-111.

19. Feinstein, A.J., Patel, M.B., Sanui, M., Cohn, S.M., Majetschak, M., and Proctor, K.G. (2005). Resuscitation with pressors after traumatic brain injury. J. Am. Coll. Surg. 201, 536-545.

20. Patel, M.B., Feinstein, A.J., Saenz, A.D., Majetschak, M., and Proctor, K.G. (2006). Prehospital HBOC-201 after traumatic brain injury and hemorrhagic shock in swine. J. Trauma 61, 46-56

21. Malhotra, A.K., Schweitzer, J.B., Fox, J.L., Fabian, T.C., and Proctor, K.G. (2003). Cerebral perfusion pressure directed therapy following traumatic brain injury and hypotension in swine. J. Neurotrauma 20, 827-839.

22. Curvello, V., Hekierski, H., Riley, J., Vavilala, M., and Armstead, W.M. (2017) Sex and age differences in phenylephrine mechanisms and outcomes after piglet brain injury. Pediatr. Res. 82, 108-113.

23. Talmor, D., Roytblat, L., Artru, A.A., Yuri, O., Koyfman, L., Katchko, L., and Shapira, Y. (1998). Phenylephrine-induced hypertension does not improve outcome after closed head trauma in rats. Anesth. Analg. 87, 574578.

24. Friess, S.H., Bruins, B., Kilbaugh, T.J., Smith, C., and Margulies, S.S. (2015). Differing effects when using phenylephrine and norepinephrine to augment cerebral blood flow after traumatic brain injury in the immature brain. J. Neurotrauma 32, 237-243.

25. Friess, S.H., Smith, C., Kilbaugh, T.J., Frangos, S.G., Ralston, J., Helfaer, M.A and Margulies, S.S. (2012). Early cerebral perfusion pressure augmentation with phenylephrine after traumatic brain injury may be neuroprotective in a pediatric swine model. Crit. Care Med. 40, 2400-2406.

26. Cherian, L., Chacko, G., Goodman, J.C., and Robertson, C.S. (1999). Cerebral hemodynamic effects of phenylephrine and I-arginine after cortical impact injury. Crit. Care Med. 27, 2512-2517.

27. Dudkiewicz, M., and Proctor, K.G. (2008). Tissue oxygenation during management of cerebral perfusion pressure with phenylephrine or vasopressin. Crit. Care Med. 36, 2641-2650.

28. Bouma, G.J., and Muizelaar, J.P. (1990). Relationship between cardiac output and cerebral blood flow in patients with intact and with impaired autoregulation. J. Neurosurg. 73, 368-374.

29. Oertel, M., Kelly, D.F., Lee, J.H., McArthur, D.L., Glenn, T.C., Vespa, P., Boscardin, W.J., Hovda, D.A., and Martin, N.A. (2002). Efficacy of hyperventilation, blood pressure elevation, and metabolic suppression therapy in controlling intracranial pressure after head injury. J. Neurosurg. 97, 1045-1053.

30. Sahuquillo, J., Amoros, S., Santos, A., Poca, M.A., Valenzuela, H., Báguena, M., and Garnacho, A. (2000). False autoregulation (pseudoautoregulation) in patients with severe head injury. Its importance in CPP management, in: A.D., Mendelow, A. Baethmann, Z. Czernicki, J.T. Hoff, U. Ito, H.E. James, T. Kuroiwa, A. Marmarou, L.F. Marshall, and H.-J. Reulen (eds). Brain Edema XI. Springer: Vienna, pps. 485-490.

31. Peterson, E., and Chesnut, R.M. (2009). Static autoregulation is intact in majority of patients with severe traumatic brain injury. J. Trauma 67, 944 949.

32. Zuercher, P., Groen, J.L., Aries, M.J.H., Steyerberg, E.W., Maas, A.I.R., Ercole, A., and Menon, D.K. (2016). Reliability and validity of the therapy intensity level scale: analysis of clinimetric properties of a novel approach to assess management of intracranial pressure in traumatic brain injury. J. Neurotrauma 33, 1768-1774.

33. Donnelly, J., Czosnyka, M., Adams, H., Cardim, D., Kolias, A.G., Zeiler, F.A Lavinio, A., Aries, M., Robba, C., Smielewski, P., Hutchinson, P.J.A., Menon, D.K., Pickard, J.D., and Budohoski, K.P. (2019). Twenty-five years of intracranial pressure monitoring after severe traumatic brain injury: a retrospective, single-center analysis. Neurosurgery 85, E75-E82.

34. Hawryluk, G.W.J., Aguilera, S., Buki, A., Bulger, E., Citerio, G., Cooper, D.J., Arrastia, R.D., Diringer, M., Figaji, A., Gao, G., Geocadin, R., Ghajar, J., Harris, O., Hoffer, A., Hutchinson, P., Joseph, M., Kitagawa, R., Manley, G., Mayer S., Menon, D.K., Meyfroidt, G., Michael, D.B., Oddo, M., Okonkwo, D., Patel, M., Robertson, C., Rosenfeld, J.V., Rubiano, A.M., Sahuquillo, J., Servadei, F., Shutter, L., Stein, D., Stocchetti, N., Taccone, F.S., Timmons, S., Tsai, E., Ullman, J.S., Vespa, P., Videtta, W., Wright, D.W., Zammit, C., and Chesnut, R.M. (2019). A management algorithm for patients with intracranial pressure monitoring: the Seattle International Severe Traumatic Brain Injury Consensus Conference (SIBICC). Intensive Care Med. 45, 17831794.
35. Lassen, N.A. (1974). Control of cerebral circulation in health and disease. Circ. Res. 34, 749-760.

36. Sorrentino, E., Diedler, J., Kasprowicz, M., Budohoski, K.P., Haubrich, C., Smielewski, P., Outtrim, J.G., Manktelow, A., Hutchinson, P.J., Pickard, J.D., Menon, D.K., and Czosnyka, M. (2012). Critical thresholds for cerebrovascular reactivity after traumatic brain injury. Neurocrit. Care 16, 258-266.

37. Zeiler, F.A., Ercole, A., Beqiri, E., Cabeleira, M., Thelin, E.P., Stocchetti, N., Steyerberg, E.W., Maas, A.I.R., Menon, D.K., Czosnyka, M., Smielewski, P., Anke, A., Beer, R., Helbok, R., Bellander, B.-M., Nelson, D., Buki, A., Chevallard, G., Citerio, G., Chieregato, A., Czeiter, E., Depreitere, B., Eapen, G. Frisvold, S., Jankowski, S., Kondziella, D., Koskinen, L.-O., Meyfroidt, G., Moeller, K., Piippo-Karjalainen, A., Raj, R., Radoi, A., Sahuquillo, J., Ragauskas, A., Rocka, S., Rhodes, J., Rossaint, R., Stevanovic, A., Sakowitz, O., Sundström, N., Takala, R., Tamosuitis, T., Tenovuo, O., Vajkoczy, P., Vargiolu, A., Vilcinis, R., Wolf, S., and Younsi, A. (2019). Association between cerebrovascular reactivity monitoring and mortality is preserved when adjusting for baseline admission characteristics in adult traumatic brain injury: a CENTER-TBI study. J. Neurotrauma 37, 12331241.

38. Bennis, F.C., Teeuwen, B., Zeiler, F.A., Elting, J.W., van der Naalt, J., Bonizzi, P., Delhaas, T., and Aries, M.J. (2020). Improving prediction of favourable outcome after 6 months in patients with severe traumatic brain injury using physiological cerebral parameters in a multivariable logistic regression model. Neurocrit. Care, doi: 10.1007/s12028-020-00930-6 [Epub ahead of print].

39. Odekon, L., Landau, R., Blouin, J.-L., Brodow, D., Wang, S., and Smiley, R.M (2015). The effect of $\beta 2$-adrenoceptor genotype on phenylephrine dose administered during spinal anesthesia for cesarean delivery. Anesth. Analg. 120, 1309-1316.

40. Hundley, W.G., Renaldo, G.J., Levasseur, J.E., and Kontos, H.A. (1988) Vasomotion in cerebral microcirculation of awake rabbits. Am. J. Physiol. 254, H67-H71.

41. Halpern, W., and Osol, G. (1985). Influence of transmural pressure of myogenic responses of isolated cerebral arteries of the rat. Ann. Biomed. Eng. 13, 287-293.

42. Auer, L.M., Ishiyama, N., and Pucher, R. (1987). Cerebrovascular response to intracranial hypertension. Acta Neurochir. (Wien) 84, 124-128.

43. Zeiler, F.A., Thelin, E.P., Donnelly, J., Stevens, A.R., Smielewski, P., Czosnyka, M., Hutchinson, P.J., and Menon, D.K. (2019). Genetic drivers of cerebral blood flow dysfunction in TBI: a speculative synthesis. Nat. Rev. Neurol. 15, 25-39.

44. Maas, A.I.R., Menon, D.K., Steyerberg, E.W., Citerio, G., Lecky, F., Manley, G.T., Hill, S., Legrand, V., Sorgner, A., and CENTER-TBI Participants and Investigators. (2015). Collaborative European NeuroTrauma Effectiveness Research in Traumatic Brain Injury (CENTER-TBI): a prospective longitudinal observational study. Neurosurgery 76, 67-80.

45. Govindan, R.B., Brady, K.M., Massaro, A.N., Perin, J., Jennings, J.M., DuPlessis, A.J., Koehler, R.C., and Lee, J.K. (2019). Comparison of frequency- and time-domain autoregulation and vasoreactivity indices in a piglet model of hypoxia-ischemia and hypothermia. Dev. Neurosci. $1-13$.

46. Zeiler, F.A., Lee, J.K., Smielewski, P., Czosnyka, M., and Brady, K. (2018). Validation of intracranial pressure-derived cerebrovascular reactivity indices against the lower limit of autoregulation, Part II: experimental model of arterial hypotension. J. Neurotrauma 35, 28122819.

47. Zeiler, F.A., Donnelly, J., Calviello, L., Lee, J.K., Smielewski, P., Brady, K., Kim, D.-J., and Czosnyka, M. (2018). Validation of pressure reactivity and pulse amplitude indices against the lower limit of autoregulation, Part I: experimental intracranial hypertension. J. Neurotrauma 35, 2803-2811.

48. Lee, J.K., Yang, Z.-J., Wang, B., Larson, A.C., Jamrogowicz, J.L., Kulikowicz, E., Kibler, K.K., Mytar, J.O., Carter, E.L., Burman, H.T., Brady, K.M., Smielewski, P., Czosnyka, M., Koehler, R.C., and Shaffner, D.H. (2012). Noninvasive autoregulation monitoring in a swine model of pediatric cardiac arrest. Anesth. Analg. 114, 825-836.

49. Brady, K.M., Lee, J.K., Kibler, K.K., Easley, R.B., Koehler, R.C., Czosnyka, M., Smielewski, P., and Shaffner, D.H. (2009). The lower limit of cerebral blood flow autoregulation is increased with elevated intracranial pressure. Anesth. Analg. 108, 1278-1283. 
50. Brady Ken M., Lee Jennifer K., Kibler Kathleen K., Easley R. Blaine, Koehler Raymond C., and Shaffner Donald H. (2008). Continuous measurement of autoregulation by spontaneous fluctuations in cerebral perfusion pressure. Stroke 39, 2531-2537.

51. Bernard, F., Gallagher, C., Griesdale, D., Kramer, A., Sekhon, M., and Zeiler, F.A. (2020). The CAnadian High-Resolution Traumatic Brain Injury (CAHR-TBI) Research Collaborative. Can. J. Neurol. Sci. J. Can. Sci. Neurol., $1-6$.

Cite this article as: Froese, L, Dian, J, Gomez, A, Unger, B, and Zeiler, FA (2020) Cerebrovascular response to phenylephrine in traumatic brain injury: A scoping systematic review of the human and animal literature, Neurotrauma Reports 1:1, 46-62, DOl:10.1089/ neur.2020.0008.

\section{Abbreviations Used}

$\mathrm{CBF}=$ cerebral blood flow

$\mathrm{CBV}=$ cerebral blood volume

$\mathrm{CPP}=$ cerebral perfusion pressure

$\mathrm{CT}=$ computed tomography

$\mathrm{CVR}=$ cerebrovascular resistance

$\mathrm{GCS}=$ Glasgow Coma Scale

$\mathrm{ICP}=$ intracranial pressure

MAP $=$ mean arterial pressure

$\mathrm{PE}=$ phenylephrine

PRISMA = Preferred Reporting Items for Systematic Reviews and Meta-Analyses

$\mathrm{PRx}=$ pressure reactivity index

$\mathrm{TBI}=$ traumatic brain injury

\section{Publish in Neurotrauma Reports}

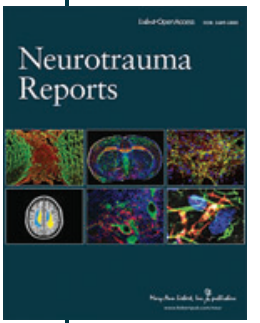

- Immediate, unrestricted online access

- Rigorous peer review

- Compliance with open access mandates

- Authors retain copyright

- Highly indexed

- Targeted email marketing

liebertpub.com/neur 